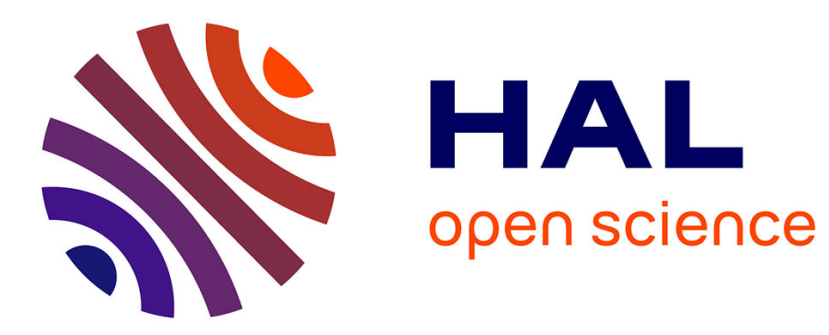

\title{
Experimental Evidence of a Rayleigh-Plateau Instability in Free Falling Granular Jets
}

\author{
Gaël Prado, Yacine Amarouchene, Hamid Null Kellay
}

\section{To cite this version:}

Gaël Prado, Yacine Amarouchene, Hamid Null Kellay. Experimental Evidence of a Rayleigh-Plateau Instability in Free Falling Granular Jets. Physical Review Letters, 2011, 106 (19), pp.198001. 10.1103/PhysRevLett.106.198001 . hal-00608839

\section{HAL Id: hal-00608839 \\ https://hal.science/hal-00608839}

Submitted on 15 Dec 2017

HAL is a multi-disciplinary open access archive for the deposit and dissemination of scientific research documents, whether they are published or not. The documents may come from teaching and research institutions in France or abroad, or from public or private research centers.
L'archive ouverte pluridisciplinaire HAL, est destinée au dépôt et à la diffusion de documents scientifiques de niveau recherche, publiés ou non, émanant des établissements d'enseignement et de recherche français ou étrangers, des laboratoires publics ou privés. 


\title{
Experimental Evidence of a Rayleigh-Plateau Instability in Free Falling Granular Jets
}

\author{
G. Prado, Y. Amarouchene, ${ }^{*}$ and H. Kellay \\ Université Bordeaux 1, Laboratoire Ondes et Matière d'Aquitaine, CNRS UMR 5798. \\ 351 cours de la libération, 33405 Talence, France
}

(Received 30 September 2010; revised manuscript received 9 March 2011; published 10 May 2011)

\begin{abstract}
A granular jet falling out of a funnel shaped container, subjected to small vertical vibrations, develops an instability farther downstream as may happen for ordinary liquid jets. Our results show that this instability is reminiscent of the Rayleigh-Plateau capillary instability leading to breakup of the jet at large scales. The first stages of this instability are captured in detail allowing a determination of the dispersion relation. Surface tensions measured in this unstable regime (of the order of $\mathrm{mN} / \mathrm{m}$ ) are in agreement with previously reported measurements carried out at much smaller scales. This instability and the breakup of the jet can be inhibited when the effect of the surrounding medium (air) is reduced by enclosing the jet in an evacuated chamber, showing that the effective surface tension measured is the result of a strong interaction with the surrounding air.
\end{abstract}

DOI: 10.1103/PhysRevLett.106.198001

PACS numbers: 45.70. $-\mathrm{n}, 47.20 . \mathrm{Dr}, 47.50 . \mathrm{Gj}$

Granular jets falling under the action of gravity display a strikingly liquidlike appearance $[1,2]$. Such jets start out spatially uniform and breakup into clusters farther downstream as may happen for ordinary liquids under the action of surface tension forces. This is surprising since attractive forces between grains (at the heart of capillarity in liquids [3]) are much weaker than other mechanical forces at play (gravity, friction, inelasticity) $[4,5]$. Such a clustering instability has been observed for free falling granular jets in air, in quiescent liquid baths, and in vacuum [6-9]. Recently, it has been shown that weak cohesive forces between grains (due to capillary bridges or van der Waals interactions) are actually sufficient for the clustering to occur [9]. This latter study pointed out that the wavelength of the clustering observed is much smaller than the one expected from a simple Rayleigh-Plateau instability, suggesting that the breakup of granular jets is difficult to reconcile with a simple capillary instability. On the other hand, the concept of an effective surface tension has been introduced in the context of granular physics by Duran in a study of the analogies between tapped granular heaps and liquid drops [10]. An effective surface tension has also been measured recently in free falling granular jets from an analysis of the statics and the dynamics of short wavelength height fluctuations at scales smaller than the jet perimeter [11]. This effective surface tension was attributed to a strong interaction of the jet with the surrounding air. If such capillary effects have been shown to be present at small scales, large scale capillary effects are unknown so far and are rather believed to be absent [12].

This Letter presents experimental results from a study of dry granular jets falling from a funnel subjected to small vertical vibrations. These jets were chosen in such a way that cohesive effects are very weak so clustering of the type seen in [9] is basically absent as seen in Fig. 1. From an analysis of the long-wavelength variations of the jet radius, it turns out that these modes are unstable and produce a long-wavelength breakup of the jet. This breakup is different from the clustering observed previously. The dispersion relation measured here from an examination of the growth rates of the imposed perturbations is in agreement with that of the "Rayleigh-Plateau" capillary instability. Our study suggests that two different breakup mechanisms operate in granular jets: one due to cohesion producing small wavelength clusters and another due to capillarity and producing breakup at larger wavelengths.

Our experimental apparatus consists of an open-topped funnel, of diameter $D$, filled with spherical glass beads (mean diameter $d=159 \mu \mathrm{m} \pm 20 \mu \mathrm{m}$ and density $\rho_{B}=$ $2500 \mathrm{~kg} \mathrm{~m}^{-3}$ ). The funnel was fixed to an electromagnetic shaker and vibrated sinusoidally with an excursion $A \sin (2 \pi f t)$ of amplitude $A$ and frequency $f$. A reduced peak acceleration, $\Gamma=A(2 \pi f)^{2} / g\left(g=9.81 \mathrm{~m} \mathrm{~s}^{-2}\right.$ is the gravitational acceleration) can easily be fixed. The mean velocity $V_{0}$ of the grains at the exit of the vertically vibrating funnel was measured using either flux measurements or PIV (particle imaging velocimetry) with $V_{0}$ in the range $0.25-0.38 \mathrm{~m} \mathrm{~s}^{-1}$ depending on the funnel used. The jet is back illuminated using a broad and diffuse white light. The position of the interface $H(z)$, or width of the jet, is measured accurately, at subpixel resolution, following the same procedure previously described in detail in [11]. Sufficient spatial and temporal resolution were achieved using a fast camera (with a $2560 \times 1600$ pixels sensor), a large number of frames (up to 40000 ) and high magnification objectives (pixel size $\sim 20 \mu \mathrm{m}$ ). To avoid electrostatic effects, we used an antistatic gun and sometimes grounded the whole set up after coating the interior of the funnel with a layer of conducting material. In addition, we tested the results using different materials for the funnel.

Figure 1(a) displays different snapshots of the granular jets, in the absence of vibration, which show a smooth and 


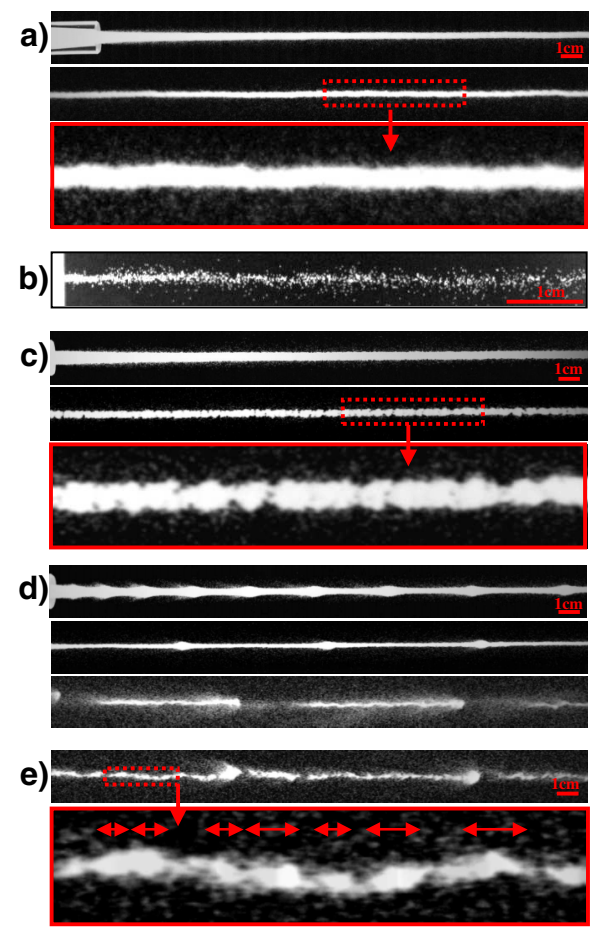

FIG. 1 (color online). (a) Jet flowing from a $D=5.3 \mathrm{~mm}$ glass funnel for (from top to bottom) $0<z<20 \mathrm{~cm}, 40<z<60 \mathrm{~cm}$ and a close up of the dashed zone for $z=55 \mathrm{~cm}$. (b) jet from a small funnel with $D / d=6.5$. (c) Jet in the same conditions as a), with addition of mineral oil ( $\sim 10^{-5}$ parts in volume), the close up shows the appearance of small clusters. (d) Modulated jet $(f=53 \mathrm{~Hz}, A=50 \mu \mathrm{m})$ for $0<z<20 \mathrm{~cm}, 40<z<60 \mathrm{~cm}$, and breakup of the jet at large scales for $100<z<120 \mathrm{~cm}$; note that the clusters present a front end asymmetry. (e) breakup of the modulated jet (same as in c) with added oil and a close up showing the small clusters.

dense flow of grains out of the funnel. Such a smooth flow is obtained for $D / d>15$, otherwise the flow of grains is discrete like [Fig. 1(b)] with clearly distinguishable grains falling under the action of gravity. For the jets used here with large $D / d$, no apparent clustering is observed up to distances of at least $100 \mathrm{~cm}$ from the outlet of the funnel. In order to generate clustering, we had to reduce the grain size to less than $100 \mu \mathrm{m}$. These observations suggest that the grains used here do not possess sufficient cohesion to produce clusters (as shown by [9] for large diameter grains or rough grains). In order to produce clusters using the $159 \mu \mathrm{m}$ grains a very small quantity of oil had to be added to the grains. Figure 1(c) shows that clusters can now be readily observed showing, in agreement with previous observations [9], that attractive forces between grains, through the presence of capillary bridges in this case, are an essential mechanism for clustering in granular media.

Figure 1(d) displays a snapshot of a vibrated granular jet. Vibration clearly generates long-wavelength modes along the jet. The wavelength of these modes increases with the distance from the exit of the funnel. The question we will examine is the following: does this modulation grow?
And if it does, can the jet breakup due solely to this growth? Figure 1(d) shows the consequences of imposing a large wavelength modulation on the jet radius. As this modulation propagates downstream it leads to the breakup of the jet with a wavelength much larger than the circumference. These observations suggest the presence of two different mechanisms leading to jet breakup: one due to cohesion between grains as previously known and another to capillarity and occurring at much larger wavelengths. In fact, when cohesion is present, the vibrated jet breaks up with two distinct scales, a small scale due to cohesion coexisting with a larger scale breakup as shown in Fig. 1(e). In the following we examine this second mode in detail using dry jets and focus on a regime for which the initial perturbation imposed to the mean profile of the jet remains small $(<5 \%)$ by analogy with liquid jets [2]. The purpose of the study is to measure the growth rate of the imposed modulations and to compare them to what is known for simple liquid jets.

The mean profile of the jet is measured for different experimental conditions and is shown in Fig. 2(a). The jet thins as it propagates downstream and its profile can be approximated by $\langle H(z)\rangle=D\left(1+2 g z / V_{0}^{2}\right)^{-1 / 4}$. This profile is independent of the frequency $f$ in the small amplitude regime suggesting that the granular jets remain incompressible even under vibration $[9,11]$. We estimate the volume fraction $\phi$ at the exit of the funnel using mass conservation through the relation $\phi=4 Q /\left(\rho_{B} V_{0} \pi D^{2}\right)$, with $Q$ the time-averaged flux. The jet density $\rho$ is then simply $\rho=\rho_{B} \phi$. The volume fraction is almost constant along the jet with $\phi \sim 0.5$ [11]. As noted in Fig. 1(c), the wavelength of the perturbation $\lambda(z)$ grows along the jet axis. This is due to the stretching imposed by the gravity field (with $V(z)=\sqrt{\left.2 g z+V_{0}^{2}\right)}$ ) coupled to the incompressibility of the jet [2]. In this case, the growth of the wavelength is simply given by $\lambda(z)=\lambda_{0}\left(1+2 g z / V_{0}^{2}\right)^{1 / 2}$ with $\lambda_{0}=V_{0} / f$. This is in good agreement with our measurements of the wavelength versus distance $z$ displayed in Fig. 2(a) for three different frequencies.

Let us focus on the spatial and temporal evolution of the perturbation induced by the vibration. Since the jet radius, the velocity of the jet, and as shown before, the effective surface tension change with vertical position $z$ [11], we use small windows in $z$ to minimize such changes. A spatiotemporal diagram of $H$ is displayed in Fig. 2(b) for $19.5 \mathrm{~cm}<z<21.5 \mathrm{~cm}$. Note that single crests are lined up along inclined straight lines and are therefore simply advected by the flow at a constant velocity $V=2 \mathrm{~m} / \mathrm{s}$, similar to the free fall velocity at this vertical location. Since the evolution of the perturbation is followed along $z$, the existence of a well defined advection velocity is necessary to convert spatial scales to temporal ones and will allow us later to investigate the mean temporal growth of the relative perturbation. The power spectrum of the temporal evolution of $H$ at a position $z=20 \mathrm{~cm}$ shows that the 


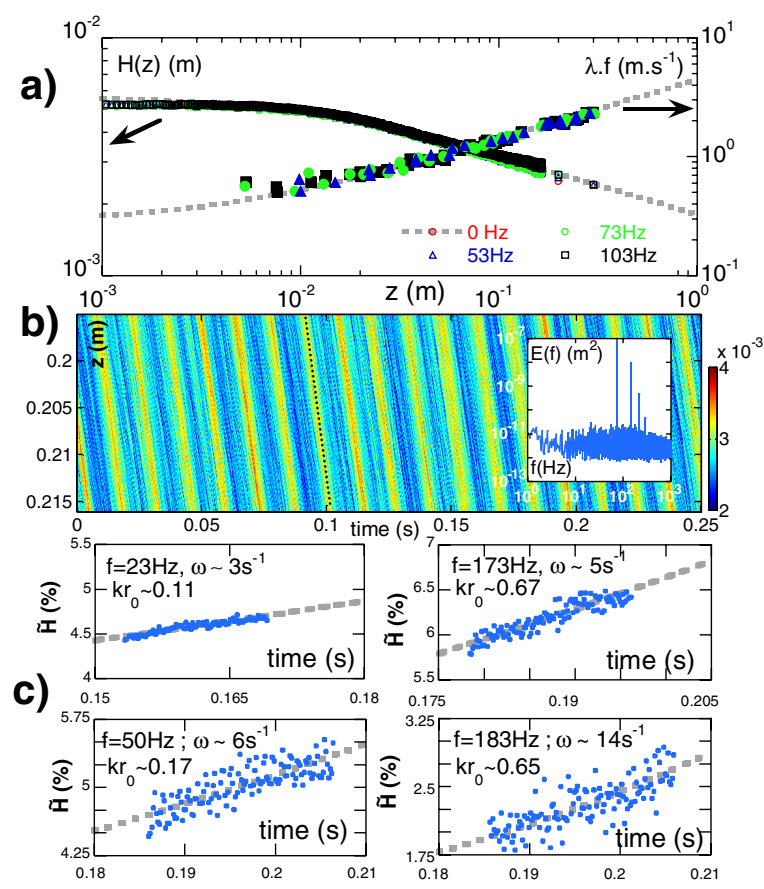

FIG. 2 (color online). (a) $\langle H(z)\rangle$ and $\lambda(z) f$ for different frequencies versus vertical distance. (b) Spatiotemporal diagram of $H$ for $f=73 \mathrm{~Hz} \& A=15 \mu \mathrm{m}$, inset: power spectrum $E(f)$. (c) $\langle\tilde{H}\rangle$ for two funnels (top panels: glass $D=5.3 \mathrm{~mm}$ funnel, $21<z<25 \mathrm{~cm}$, bottom panels polypropylene $D=6.3 \mathrm{~mm}$ funnel, $23<z<27 \mathrm{~cm}$ ).

amplitude of the excited mode is important, see inset of Fig. 2(b). Along with this fundamental excitation mode, other harmonics are present (modes $2 f$ and $3 f$ ). Thereby, a pure peristaltic mode is not present. However, since the amplitudes of the high order harmonics are small (more than an order of magnitude), we neglect them in our analysis and focus only on the amplitude of the imposed frequency, which amounts to band-pass filtering the signal at this frequency. For all the experiments presented here, the non dimensionalized acceleration $\Gamma$ was kept small and typically less than 1 to avoid the excitation of subharmonics.

A quantity of interest is the relative amplitude of the perturbation given by $\tilde{H}=\frac{H-\langle H\rangle}{\langle H\rangle}$ which allows us to decouple the evolution of the perturbation from the mean thinning of the jet. The temporal evolution of the averaged value $\langle\tilde{H}\rangle$ (measured using the amplitude extracted from the spectral analysis at the frequency of excitation) is displayed in Fig. 2(c) for different frequencies and two different jet diameters. Vertical spatial scales $z$ are converted to temporal ones as $t=\left(V(z)-V_{0}\right) / g$. The temporal growth rates $\omega$ extracted from exponential fits to the data are positive and point to the existence of an amplification of the initial perturbation. The order of magnitude of the growth rates measured is a few $s^{-1}$. The wavelength of the perturbation $\lambda$ is always larger than the perimeter of the jet which corresponds to the long-wavelength limit with reduced wave number $k r_{0}<1$ ( $k=\frac{2 \pi}{\lambda}$ with $r_{0}$ the mean radius of the jet). Note that the growth rate changes with frequency and increases as the jet radius decreases. Additional measurements, where the evolution of the amplitude of single crests was determined, were performed. While the data for the growth rates are more scattered from crest to crest (due to poorer statistics), the mean values extracted from an ensemble average are in good agreement with the previous global estimate.

The growth rates $\omega$ versus $k r_{0}$ extracted from several experiments using several frequencies and for $19.5 \mathrm{~cm}<$ $z<22.5 \mathrm{~cm}$ downstream from the outlet are shown in Fig. 3(a). Despite the large scatter of the data, a bell shaped curve with a maximum occurring around $k r_{0} \sim 0.7$ is observed for the glass funnel. Data for two other jet diameters obtained using plastic funnels (the excitation of high frequency modes was more difficult to achieve with the plastic funnels) are shown and clearly indicate a strong dependence on the radius of the jet. These observations are reminiscent of a Rayleigh-Plateau instability. Indeed, in the inviscid limit of this instability, modes with wavelengths larger than the jet circumference $2 \pi r_{0}$ are unstable and are simply advected by the mean flow $[2,13]$. The wellknown dispersion relation which relates the growth rate of the perturbation to the wave number, is given by $\omega^{2}=\frac{\gamma}{\rho r_{0}^{3}} k r_{0} \frac{I_{1}\left(k r_{0}\right)}{I_{0}\left(k r_{0}\right)}\left[1-\left(k r_{0}\right)^{2}\right]$. Here $\gamma$, and $I_{n}$ are, respectively, the surface tension and the modified Bessel function of order $n[2,13]$. A fit to the data using this dispersion relation is displayed in Fig. 3(a). The agreement with the experimental data is very good for the three different jet diameters.

Since $r_{0}, \rho$, and $k$ are measured independently, the only unknown parameter is the surface tension $\gamma$ which can be extracted from a single parameter fit. It turns out that $\gamma$ is of the order of a few $\mathrm{mN} / \mathrm{m}$ and depends barely on the funnel diameter used. Since the effective surface tension has been shown to depend on the vertical location $z$ [11], we have examined the growth rate of the imposed perturbations in other small windows centered at different locations $z$. The results of these experiments are shown in the inset of Fig. 3(a) where the surface tension extracted from the growth rate is compared to that measured at the specific location $z$ using the method proposed in [11]. The agreement is good suggesting that the effective surface tension obtained from an analysis at small scales is similar to that extracted from the large scale analysis carried out here.

As mentioned in the beginning of this Letter, granular jets breakup under the effect of cohesion or weak attractive forces between grains into clusters separated by a scale which is smaller than expected from a Rayleigh-Plateau like instability. Typically, the scale of breakup of liquid jets under the action of a Rayleigh-Plateau instability is around the maximum of the dispersion curve, i.e., around $k r_{0}=0.7$, or smaller. Observations of clustering in granular jets find values of $k r_{0}$ distributed between 1 and 3 which 

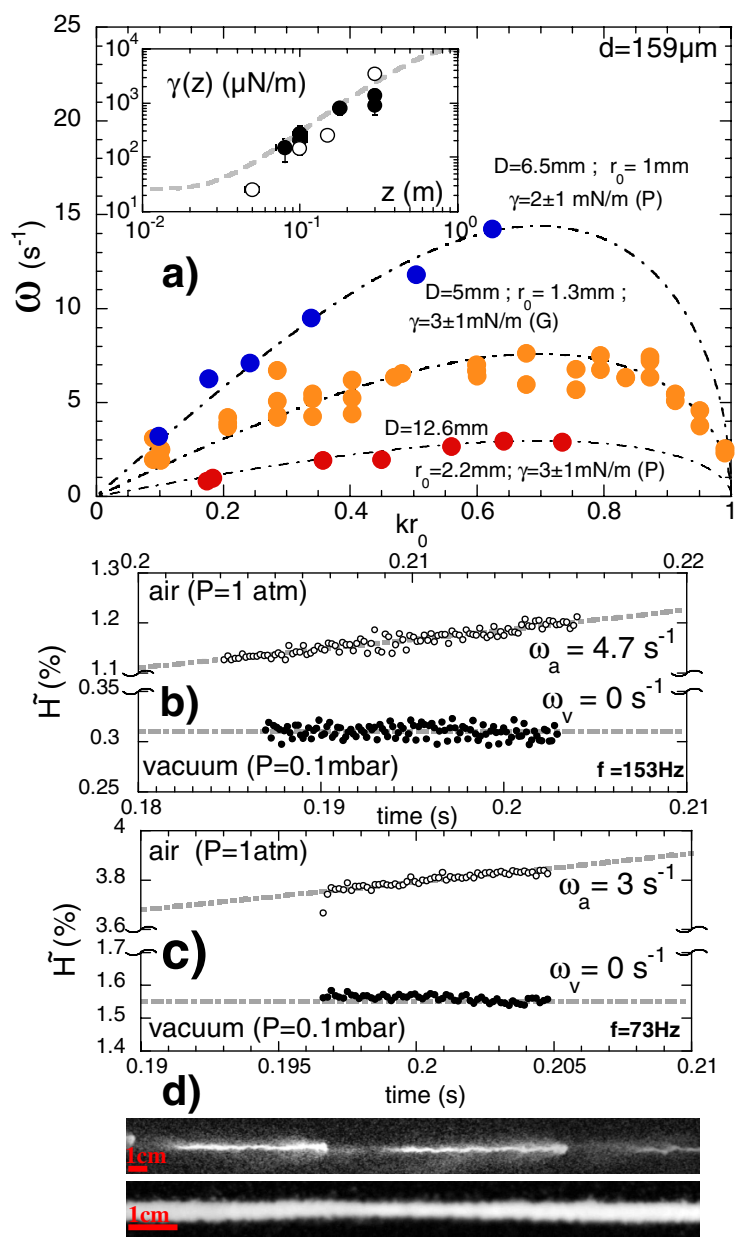

FIG. 3 (color online). (a) Dispersion relations $\omega\left(k r_{0}\right)$ for $z$ centered at $20 \mathrm{~cm}$ for three funnels [glass $(\mathrm{G})$ or plastic (Polypropylene), $V_{0}=0.25 \mathrm{~m} \mathrm{~s}^{-1}$ for plastic, $V_{0}=0.38 \mathrm{~m} \mathrm{~s}^{-1}$ for glass]. Inset: surface tension extracted from long-wavelength modes (black circles) and small scale analysis (white circles). The gray line is a guide to the eye. (b)-(c) Comparison of growth rates at ambient pressure and in vacuum $(0.1 \mathrm{mbar})$ for $D=8.3 \mathrm{~mm}$. (d) Comparison of a modulated jet ( $f=53 \mathrm{~Hz}$, $A=50 \mu \mathrm{m}$ ) in air at ambient pressure (top image) for $100<z<120 \mathrm{~cm}$ and a modulated jet $(f=53 \mathrm{~Hz}$, $A=100 \mu \mathrm{m}$ ) at a pressure of $0.08 \mathrm{mbar}$ (bottom image) for $100<z<110 \mathrm{~cm}$ showing no breakup.

basically puts clustering in a regime where perturbations should not grow via such an instability. For the noncohesive grains used here, breakup may be achieved at smaller values of $k r_{0}$ and always less than 1 even though the growth rates are weak. This observation suggests that different mechanisms operate in the breakup of granular jets: one due to cohesion and another due to a capillary instability. Capillary effects have been attributed to the interaction of the jet with the surrounding air. Such an effect should therefore be absent in vacuum. By enclosing the whole set up in a sealed and evacuated plastic tube ( $9 \mathrm{~cm}$ in diameter and $150 \mathrm{~cm}$ long) we were able to study the effect of air pressure on the stability of such longwavelength modes. Figure 3(b) and 3(c) show the temporal variation of the relative amplitude of an excited mode both in air and in an evacuated chamber at a pressure of 0.1 mbar. In air (in the cylindrical chamber at a pressure of $1 \mathrm{~atm}$.) the growth rate is positive and in agreement with those shown in Fig. 2(c) and 3(a). In the evacuated chamber, however, the growth rate vanishes and the breakup of the jet is inhibited even far away from the entrance as shown in Fig. 3(d). The effect of the ambient air is therefore crucial for the development of a capillarylike instability of granular jets leading to their breakup at large scales.

In conclusion, our results show that capillary effects leading to the well-known Rayleigh-Plateau instability can be observed for granular jets falling in air. The first stages of this instability are captured in detail through the complete measurement of the dispersion relation which turns out to be well described by the predictions of the inviscid linear stability theory of Rayleigh valid for small viscosity liquid columns. Surface tensions estimated in this unstable regime are in agreement with previously reported measurements carried out at small scales, suggesting that a single surface tension exists for a wide range of scales in a falling granular jet. These observations provide a second route to breaking of granular jets along with the recently observed clustering due to cohesion and liquid bridges between grains.

This research is supported by CR Aquitaine Grants no. 2006111101035, no. 20091101004 and by ANR Grant no. ANR-09-JCJC-0092.

*y.amarouchene@loma.u-bordeaux1.fr

[1] N. Khamontova, J. Russ. Phys. Chem. Soc. 22, 281 (1890).

[2] J. Eggers and E. Villermaux, Rep. Prog. Phys. 71, 036601 (2008).

[3] J.S. Rowlinson, B. Widom, Molecular Theory of Capillarity (Dover, New York, 2002).

[4] H. M. Jaeger, S. R. Nagel, and R. P. Behringer, Rev. Mod. Phys. 68, 1259 (1996).

[5] I. S. Aranson and L. S. Tsimring, Rev. Mod. Phys. 78, 641 (2006).

[6] U. Schaflinger and G. Machu, Chem. Eng. Technol. 22, 617 (1999).

[7] M. Nicolas, Phys. Fluids 14, 3570 (2002).

[8] M. E. Möbius, Phys. Rev. E 74, 051304 (2006).

[9] J. R. Royer et al., Nature (London) 459, 1110 (2009).

[10] J. Duran, Phys. Rev. Lett. 87, 254301 (2001).

[11] Y. Amarouchene, J. F. Boudet, and H. Kellay, Phys. Rev. Lett. 100, 218001 (2008).

[12] X. Cheng et al., Phys. Rev. Lett. 99, 188001 (2007).

[13] S. Chandrasekhar, Hydrodynamic and Hydromagnetic Stability (Dover, New York, 1961). 